\title{
Editorials
}

\section{Primary care networks:}

\author{
are they fit for the future?
}

\section{INTRODUCTION}

There are 1250 primary care networks (PCNs) in the English NHS. Established in July 2019, PCNs have been developed in response to national policy focused on better integrating health and social care services while strengthening the sustainability of primary care. ${ }^{1}$ They receive additional funding to support the employment of new types of practitioners and provide enhanced services to patients. While originally intended to cover populations of 30000 to 50000 patients, only $58 \%$ of PCNs are within this range, with some smaller, and more that are larger. ${ }^{2}$

\section{RESEARCH EVIDENCE SO FAR}

Research into the implementation and early development of PCNs suggests swift and impressive progress compared with some of their antecedent primary care organisations, with initial governance arrangements in place, and staff recruited to deliver practice-based pharmacy, social prescribing, and enhanced support for care homes. ${ }^{3}$ PCNs are also playing an important role in primary care responses to the pandemic ${ }^{3}$ and the COVID-19 vaccination programme. ${ }^{4}$

This seemingly positive and striking early progress conceals a profound organisational fragility that emerged as an important theme in the two National Institute for Health Research funded studies of PCNs., ${ }^{3,5}$ First, much is expected of these new networks. They are viewed as important routes through which to channel new resources to strengthen local primary care services, and as a lynchpin of the NHS Long Term Plan ambitions towards better integrated health and social care, with services based outside hospital wherever possible. ${ }^{6}$ PCNs are intended to deliver many new services, as indicated in

\section{early evaluation studies suggest that primary care networks (PCNs) are operating on something of a shoestring. Unlike predecessor primary care organisations ... PCNs do not have defined per capita fincancial allocations for management costs.}

the NHS England service specifications.? Furthermore, recently published proposals for integrated care systems in England imply an expanded role for PCNs in coordinating local primary care provision and representing primary care at system level. These expectations are occurring in a context of likely mergers or abolition of the clinical commissioning groups (CCGs) that to date have developed and supported PCNs.

Second, our early evaluation studies 3,5 suggest that PCNs are operating on something of a shoestring. Unlike predecessor primary care organisations such as practice-based commissioning or primary care groups, PCNs do not have defined per capita financial allocations for management costs. Much therefore rests on PCN clinical directors, who can claim just 0.25 FTE of back-fill funding per 50000 patients for their work in leading and managing the PCN. ${ }^{5}$ Some have resigned, or only committed to the role for short periods, due to the unmanageable workload. ${ }^{3,5}$ Other management support has to be secured from local practices, preexisting primary care collaborations such as super-partnerships and federations, CCGs, or a local NHS trust

Third, if PCNs are to assume a significant role within integrated care systems, contribute to mooted provider

\section{"As we contemplate 2021, primary care networks face the challenge of leading and supporting local general practice teams through the remaining phases of the COVID-19 pandemic, and meeting the additional needs of patients and their families in a challenging post- pandemic context.}

collaboratives', ${ }^{8}$ and develop services collaboratively with other practitioners such as pharmacists, dentists, and the third sector, they will likely need additional management and professional expertise including: project management support; population health needs analysis; organisational development; financial management; and human resources and change management. At present, there is no indication as to where the funding for such support may come from, nor that this need has been recognised in national policy.

\section{POLICY IMPLICATIONS}

General practice collaborations have been a feature of the NHS and other health systems for over three decades, with 'at-scale' primary care seen increasingly as a way of enabling local service development, supporting practices, and giving primary care a stronger 'voice' in the local health system. ${ }^{9}$ Studies across the years have shown the vital importance of sufficient, high-quality management and organisational support for these primary care collaborations, with such capacity having a direct influence on the progress that can be made. ${ }^{10,11}$ Good support from local primary care commissioners has been shown to be vital, ${ }^{9}$ and it is concerning that current NHS England and Improvement proposals ${ }^{8}$ for system change apparently do not include a role for locally-based primary care commissioning and planning authorities, beyond the representation of PCNs within integrated care system governance boards.

As we contemplate 2021, PCNs face the challenge of leading and supporting local general practice teams through the remaining phases of the COVID-19 pandemic, and meeting the additional needs of patients and their families in a challenging post-pandemic context. On top of this, they will have to navigate a likely NHS reorganisation as integrated care systems 


\section{"Primary care networks will need sufficient management, leadership capacity, and expertise to enable them to survive and thrive in these likely turbulent times."}

are established, and primary care seeks to ensure a strong voice. PCNs will need sufficient management, leadership capacity, and expertise to enable them to survive and thrive in these likely turbulent times. How far they will be supported to be fit for this future lies in the hands of NHS England and Improvement, and those who commission primary care.

Perhaps the most urgent task lies in supporting PCNs to develop the capacity to look beyond the immediate crisis to consider how they can most effectively collaborate with community and other providers across geographical areas to better integrate care and support improvements in population health.

\section{Judith A Smith,}

Director and Professor of Health Policy and

Management, Health Services Management Centre, University of Birmingham, Birmingham.

\section{Katherine Checkland,}

Professor of Health Policy and Primary Care, Division of Population Health, Health Services Research and Primary Care, University of

Manchester, Manchester.

\section{Manbinder Sidhu,}

Research Fellow, Health Services Management Centre, University of Birmingham, Birmingham.

\section{Jonathan Hammond,}

Research Fellow, Division of Population Health, Health Services Research and Primary Care, University of Manchester, Manchester.

\section{Sarah Parkinson,}

Analyst, RAND Europe, Cambridge.

\section{Funding}

Some of the research on which this article draws was carried out by the Birmingham, RAND and Cambridge Evaluation Centre (BRACE), which is funded by the National Institute for Health Research (NIHR), Health Services and Delivery Research programme (reference: HSDR 16/138/31). Further research on which this article draws was carried out by the Policy Research Unit in the Health and Care System and Commissioning (PRUComm), funded by the NIHR Policy Research Programme (reference: PR-PRU-1217-20801). The views expressed are those of the authors and not necessarily those of the NIHR, the Department of Health and Social Care, or the Policy Research Programme.

\section{Provenance}

Commissioned; externally peer reviewed.

\section{Competing interests}

The authors have declared no competing interests.

DOI: https://doi.org/10.3399/bjgp21X714665

\section{EMAIL ADDRESS FOR CORRESPONDENCE}

\section{Judith A Smith}

Email: j.a.smith.20囚bham.ac.uk

\section{REFERENCES}

1. Baird B, Beech J. Primary care networks explained. 2020. https://www.kingsfund.org. uk/publications/primary-care-networksexplained (accessed 3 Feb 2021).

2. Morciano M, Checkland $\mathrm{K}$, Hammond J, et al. Variability in size and characteristics of primary care networks in England: observational study. Br J Gen Pract 2020; DOI: https://doi.org/10.3399/bjgp20X713441.

3. Smith J, Parkinson S, Harshfield A, Sidhu M. Early evidence of the development of primary care networks in England: a rapid evaluation study. Health Services and Delivery Research 2020; DOI: https://doi.org/10.3310/hsdrtr-129678.

4. NHS England. PCN sites beginning COVID19 vaccination next week - information to support you to stand up your vaccination service. 2020. https://www.england.nhs. uk/coronavirus/wp-content/uploads/ sites/52/2020/12/C0944-pcn-sites-beginningcovid-19-vaccination-support-information.pdf laccessed 3 Feb 2021)

5. Checkland K, Hammond J, Morciano M et al. Primary care networks: exploring primary care commissioning, contracting and provision. 2020. https://prucomm.ac.uk/ assets/uploads/PRU-PCN_interim_report_ FINAL.pdf (accessed 3 Feb 2021)

6. NHS England. The NHS Long Term Plan. 2019. https://www.longtermplan.nhs.uk/ wp-content/uploads/2019/08/nhs-long-termplan-version-1.2.pdf laccessed 3 Feb 2021).

7. NHS England. Network Contract Directed Enhanced Service: contract specification 2020/21 - PCN requirements and entitlements. 2020. https://www.england. nhs.uk/wp-content/uploads/2020/03/ Network-Contract-DES-Specification-PCNRequirements-and-Entitlements-2020-21 October-FINAL.pdf laccessed 4 Feb 2021).

8. NHS England, NHS Improvement. Integrating care: next steps to building strong and effective integrated care systems across England. 2021. https://www.england.nhs.uk/ publication/integrating-care-next-steps-tobuilding-strong-and-effective-integratedcare-systems-across-england laccessed 4 Feb 2021).

9. Pettigrew L, Kumpunen S, Rosen R, et al. Lessons for 'large-scale' general practice provider organisations in England from other inter-organisational healthcare collaborations. Health Policy 2019; 123(1): 51-61.

10. Mays N, Wyke S, Malbon G, Goodwin N, eds. The purchasing of health care by primary care organizations. An evaluation and guide to future policy. Buckingham: Open University Press, 2001.

11. Miller R, Peckham $S$, Coleman $A$, et al. What happens when GPs engage in commissioning? Two decades of experience in the English NHS. J Health Serv Res Policy 2016; 21(2): 126-133. 